\title{
A note on letters of Yangian invariants
}

\author{
Song $\mathbf{H e} \mathbf{e}^{a, b, c, d}$ and Zhenjie $\mathbf{L i}^{a, d}$ \\ ${ }^{a}$ CAS Key Laboratory of Theoretical Physics, \\ Institute of Theoretical Physics, Chinese Academy of Sciences, \\ Beijing 100190, China \\ ${ }^{b}$ School of Fundamental Physics and Mathematical Sciences, \\ Hangzhou Institute for Advanced Study, \\ UCAS, Hangzhou 310024, China \\ ${ }^{c}$ ICTP-AP International Centre for Theoretical Physics Asia-Pacific, \\ Beijing/Hangzhou, China \\ ${ }^{d}$ School of Physical Sciences, University of Chinese Academy of Sciences, \\ No.19A Yuquan Road, Beijing 100049, China \\ E-mail: songhe@itp.ac.cn, lizhenjie@itp.ac.cn
}

ABSTRACT: Motivated by reformulating Yangian invariants in planar $\mathcal{N}=4 \mathrm{SYM}$ directly as $d \log$ forms on momentum-twistor space, we propose a purely algebraic problem of determining the arguments of the $d \log$ 's, which we call "letters", for any Yangian invariant. These are functions of momentum twistors $Z$ 's, given by the positive coordinates $\alpha$ 's of parametrizations of the matrix $C(\alpha)$, evaluated on the support of polynomial equations $C(\alpha) \cdot Z=0$. We provide evidence that the letters of Yangian invariants are related to the cluster algebra of Grassmannian $G(4, n)$, which is relevant for the symbol alphabet of $n$-point scattering amplitudes. For $n=6,7$, the collection of letters for all Yangian invariants contains the cluster $\mathcal{A}$ coordinates of $G(4, n)$. We determine algebraic letters of Yangian invariant associated with any "four-mass" box, which for $n=8$ reproduce the 18 multiplicative-independent, algebraic symbol letters discovered recently for two-loop amplitudes.

KeYwords: Scattering Amplitudes, Supersymmetric Gauge Theory

ArXiv EPrint: 2007.01574 


\section{Contents}

1 Introduction 1

2 Letters of Yangian invariants 3

2.1 Letters of NMHV and $\overline{\mathrm{MHV}}$ invariants 4

2.2 Letters of $n=6$ and $n=7$ invariants 6

2.3 Algebraic letters of $\mathrm{N}^{2} \mathrm{MHV}$ invariants $\quad 9$

3 Discussions 11

\section{Introduction}

Recent years have witnessed tremendous progress in unravelling hidden mathematical structures of scattering amplitudes, especially in planar $\mathcal{N}=4$ supersymmetric Yang-Mills theory (SYM) (cf. [1, 2]). Moreover, formidable progress has been made in computing multi-loop scattering amplitudes of the theory, most notably by the hexagon and heptagon bootstrap program [3-8]. A crucial assumption for the bootstrap is that the collection of the letters entering the symbol (which is the maximally iterated coproduct of generalized polylogarithms), or the alphabet, consists of only 9 and 42 cluster variables of $G(4, n)[9]$. These are dual conformally invariant (DCI), rational functions of momentum twistors describing the kinematics of $n$-point amplitudes [10]. The space of generalized polylogarithms with correct alphabet is remarkably small, and certain information from physical limits and symmetries suffices to fix the result (see [11] for a recent review). The symbol of the sixpoint amplitude (or hexagon) has been determined through seven and six loops for MHV and NMHV cases respectively [12], and similarly that of the seven-point amplitude (or heptagon) has been determined through four loops for these cases respectively $[13,14]$.

Starting $n=8$, the cluster bootstrap becomes intractable since the Grassmannian cluster algebra [15] becomes infinite type. It is an important open question how to find a finite subset of cluster variables which appear in the symbol alphabet of multi-loop amplitudes, and there has been significant progress e.g. by studying their Landau singularities [16-19]. Moreover, it is known that starting $n=8$, algebraic (irrational) functions of momentum twistors appear in the alphabet, as one can already see in four-mass box integrals needed for one-loop amplitudes with $k \geq 2$ (such algebraic letters are also present for higher-loop MHV amplitudes with $n \geq 8$ ).

Recently, a new computation for two-loop $n=8 \mathrm{NMHV}$ amplitude is performed using the so-called $\bar{Q}$ equations [20], which provides a candidate of the symbol alphabet for $n=8$; in particular, it provides the space of 18 algebraic letters which involve square roots of the 
kinematics. After that new proposals concerning the symbol alphabet for $n \geq 8$ have appeared [21-23], which exploit mathematical structures such as tropical Grassmannian [24] and positive configuration space [25].

In this short note, we make a simple observation that an alternative construction, based on solving polynomial equations associated with plabic graphs for Yangian invariants, may provide another route to symbol alphabet including algebraic letters. We will argue that the alphabet of Yangian invariants, which will be defined shortly, contains not only the (rational) symbol letters of $n=6$ and $n=7$ amplitudes but also the algebraic letters for two-loop $n=8$ NMHV amplitude mentioned above.

We emphasize that generally for any Yangian invariant, the alphabet includes but is not restricted to the collection of poles, which have been explored in the literature so far, e.g. in the context of the so-called cluster adjacency [14, 26-30]. As we will see already for $n=7$, by going through all Yangian invariants we find 42 poles in total, which are 7 frozen variables and 35 unfrozen ones, i.e. 7 of the 42 cluster $\mathcal{A}$ variables of $G(4,7)$ are still missing! On the other hand, the alphabet of all possible Yangian invariants consists of 63 letters, which include the 42 cluster variables. The search for alphabets of Yangian invariants is by itself a beautiful open problem related to positive Grassmannian, plabic graphs and cluster algebra [1], and let's first formulate the general problem as suggested by [31].

An $\mathrm{N}^{k} \mathrm{MHV}$ Yangian invariant is an on-shell function of (super-)momentum twistors $\{Z \mid \eta\}_{1 \leq a \leq n}$, which is associated with a certain $4 k$-dimensional positroid cell of the positive Grassmannian $G_{+}(k, n) ;^{1}$ the cell can be parametrized by a $k \times n$ matrix $C\left(\{\alpha\}_{1 \leq i \leq 4 k}\right)$ where $\alpha_{i}$ 's are positive coordinates, and the on-shell function (which has Grassmann-degree $4 k)$ is given by

$$
Y(\{Z \mid \eta\})=\int \prod_{i=1}^{4 k} d \log \alpha_{i} \delta^{4 k \mid 4 k}(C(\{\alpha\}) \cdot(Z \mid \eta)),
$$

where the contour of the integral encloses solutions of equations $\sum_{a=1}^{n} C_{I, a}(\{\alpha\}) Z_{a}=0$ (for $I=1,2, \cdots, k)$. As first proposed in [32] and studied further in [33], it is instructive to write Yangian invariants, or more general on-shell (super-)functions, as differential forms on momentum-twistor space, where we replace the fermionic variables $\eta_{a}$ by the differential $d Z_{a}$ for $a=1, \cdots, n$. Remarkably, after the replacement the Yangian invariant defined as a $4 k$ form, $\mathcal{Y}(\{Z \mid d Z\}):=\left.Y\right|_{\eta_{a} \rightarrow d Z_{a}}$, becomes the pushforward of the canonical form of the cell, $\prod_{i} d \log \alpha_{i}$, to $Z$ space:

$$
\mathcal{Y}^{*}(Z \mid d Z)=\left\{\bigwedge_{i=1}^{4 k} d \log \alpha_{i}^{*}(Z)\right\}, \quad \text { with }\left\{\alpha^{*}(Z)\right\} \text { solutions of } C(\alpha) \cdot Z=0 .
$$

In other words, we evaluate the form on solutions, $\alpha^{*}(Z)$, of the polynomial equations $C(\alpha) \cdot Z=0$, and we have used subscript $*$ as a reminder that there can be multiple solutions in which case $\mathcal{Y}^{*}$ becomes a collection of $4 k$ forms, one for each solution (the number of solutions, $\Gamma(C)$ is given by the number of isolated points in the intersection $\left.C^{\perp} \cap Z[1]\right)$. The solutions, $\alpha^{*}(Z)$, are GL(1)-invariant functions of Plücker coordinates on

\footnotetext{
${ }^{1}$ One can easily extend our construction to general $m$ but in this note we only consider $m=4$.
} 
$G(4, n),\langle a b c d\rangle:=\operatorname{det}\left(Z_{a} Z_{b} Z_{c} Z_{d}\right)$, and when there are multiple solutions, $\Gamma(C)>1$, they are generally algebraic functions.

Since symbol letters of loop amplitudes are related to arguments and indices of the involved generalized polylogarithms, it is only natural to refer to the arguments of the $d \log$ 's on the support of $C \cdot Z=0$, as the letters of a given Yangian invariant. One can define the alphabet of a Yangian invariant to be the collection of possible letters, i.e. the solutions $\left\{\alpha_{i}^{*}(Z)\right\}$, which form a $4 k \times \Gamma(C)$ matrix. However, there are ambiguities since under a reparametrization of the cell, $C(\{\alpha\}) \rightarrow C\left(\left\{\alpha^{\prime}\right\}\right)$, which leaves the form invariant, the alphabet generally changes. Such reparametrizations are related to cluster transformations acting on variables of (positroid cells of) $G_{+}(k, n)$ [1]. In principle one could attempt to find all possible letters for a given positroid cell, by scanning through all transformations that leave the canonical form invariant, but the resulting collection of letters can easily be infinite for $n \geq 8$ !

Therefore, we restrict ourselves to a very small subset of such transformations, namely those generated by equivalence moves acting on plabic graphs associated with a positroid cell. Therefore, we define the alphabet of any given Yangian invariant to be the collection of face or edge variables of all possible plabic graphs. It is obvious that this collection of letters is finite for any Yangian invariant, and we can further take the union of the alphabets for all Yangian invariants of the same $n$ and $k$, which will be referred to as the alphabet of a given $n$ and $k$.

There is still a residual redundancy in this definition: any monomial reparametrization of the form $\alpha_{j}^{\prime}=\prod_{i} \alpha_{i}^{n_{i, j}}$ for $1 \leq j \leq d$ with $\operatorname{det} n_{i, j}=1$ leaves the form invariant. Even for a given plabic graph, we have such redundancy in the definition of face or edge variables, and the invariant content of the alphabet is the space generated by monomials of $\left\{\alpha^{*}(Z)_{i}\right\}$ (or the linear space spanned by $\left\{\log \alpha_{i}^{*}\right\}$ ), and one can choose some representative letters which give the same space. For example, when $\alpha^{\prime}(Z)$ are rational with $\Gamma(C)=1$, one can always choose a set of irreducible polynomials of Plücker coordinates, which are analogous to $\mathcal{A}$ coordinates of cluster algebra of $G(4, n)$. Similar choices can be made for algebraic solutions with $\Gamma(C)>1$, modulo ambiguities from the fact that monomials of these algebraic letters can form rational letters which are irreducible polynomials.

In our definition, letters of Yangian invariants are generally not DCI, thus they are analogous to the cluster $\mathcal{A}$ - coordinates. It is trivial to homogenize them and obtain the analog of cluster $\mathcal{X}$ coordinates. For our main example of algebraic letters, where the Yangian invariants correspond to leading singularities of four-mass boxes, a natural way to define $\mathcal{X}$-like variables is by considering ratio of conjugates involving the square roots. We find that the collection of such ratios turns out to give precisely the same 18 algebraic alphabet for two-loop NMHV amplitudes in [34].

\section{Letters of Yangian invariants}

In the following, we will illustrate our algorithm by finding (subsets of) alphabets of Yangian invariants for certain $n$ and $k$. The procedure goes as follows: for any given $n$ and $k$, we first scan through all possible $4 k$-dimensional cell of $G_{+}(k, n)$ and list the resulting 
Yangian invariants. The representation of any Yangian invariant can be obtained using the procedure given in [1], in terms of the matrix $C(\{\alpha\})$ with canonical coordinates $\{\alpha\}_{1 \leq i \leq 4 k}$ for the cell; equivalently these coordinates can be identified with non-trivial edge variables of a representation plabic graph. Then one can start to apply square moves to the graph, which generate reparametrization of the cell and possibly find more letters. In simple cases, it is straightforward to find all equivalence moves and obtain the complete alphabet of the Yangian invariant; for more involved cases, we will content with ourselves in finding a subset of the alphabet by applying such move once, and the results turn out to be already illuminating.

\subsection{Letters of NMHV and $\overline{\mathrm{MHV}}$ invariants}

First, we present the simplest non-trivial Yangian invariant, which is the only type for NMHV $(k=1)$. A 4 -dimensional cell $C \in G_{+}(1, n)$ can be parametrized as

$$
\left(\ldots, 0,1,0 \ldots, 0, \alpha_{1}, 0, \ldots, 0, \alpha_{2}, 0, \ldots, 0, \alpha_{3}, 0, \ldots, 0, \ldots, 0, \alpha_{4}, 0, \ldots\right)
$$

where only $a, b, c, d$ and $e$-th entry are non-zero, and the on-shell function is given by the $\mathrm{R}$ invariant $[a, b, c, d, e]$. The solution of the linear equation $C \cdot Z=0$ is simply

$$
\alpha_{1}=-\frac{\langle a c d e\rangle}{\langle b c d e\rangle}, \quad \alpha_{2}=-\frac{\langle a b d e\rangle}{\langle c b d e\rangle}, \quad \alpha_{3}=-\frac{\langle a b c e\rangle}{\langle d b c e\rangle}, \quad \alpha_{4}=-\frac{\langle a b c d\rangle}{\langle e b c d\rangle},
$$

and one can easily check that $\mathcal{Y}_{n, 1}(a, b, c, d, e)=\prod_{i=1}^{4} d \log \alpha_{i}^{*}(Z)$ is indeed the form obtained by replacing $\eta$ by $d Z$ in $[a, b, c, d, e]$. Obviously any reparametrization is trivial in this case, and we can choose representative letters to be the five Plücker coordinates appeared above.

By taking the union of alphabets of all NMHV invariants for $n$ points, we trivially get the collection of all Plücker coordinates. Also note that in this special case the representative letters are exactly the poles of the Yangian invariant, but we will see immediately that this is no longer true beyond NMHV.

Our next example is for $\overline{\mathrm{MHV}}(k=n-4)$, where the unique Yangian invariant for $n$ points is given by the top cell of $G_{+}(n-4, n)$ (or equivalently that of $G_{+}(4, n)$, and the form is the familiar cyclic measure:

$$
\mathcal{Y}_{n, n-4}=\frac{d^{4 n} Z /(\operatorname{vol} \mathrm{GL}(4))}{(1234)(2345) \cdots(n 123)}
$$

The poles of the invariant ( $\overline{\mathrm{MHV}}$ amplitude) are the frozen Plücker coordinates, as they should be. However, the letters for any plabic graph involve variables other than these frozen ones. Let's start with $n=6$ (for $n=5$ it is just NMHV invariant $[1,2,3,4,5]$ ), and 
a representative plabic graph is

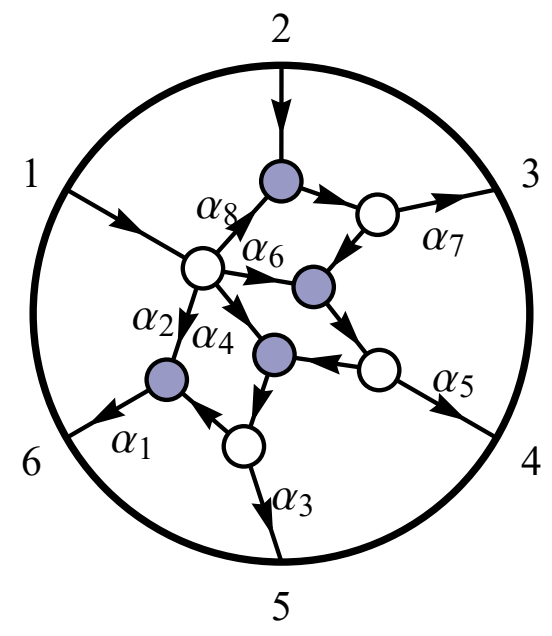

The corresponding $C$ matrix reads

$$
\left(\begin{array}{cccccc}
1 & \alpha_{2}+\alpha_{4}+\alpha_{6}+\alpha_{8} & \left(\alpha_{2}+\alpha_{4}+\alpha_{6}\right) \alpha_{7} & \left(\alpha_{2}+\alpha_{4}\right) \alpha_{5} & \alpha_{2} \alpha_{3} & 0 \\
0 & 1 & \alpha_{7} & \alpha_{5} & \alpha_{3} & \alpha_{1}
\end{array}\right)
$$

and the solution of $C(\alpha) \cdot Z=0$ is

$$
\begin{array}{llll}
\alpha_{1}=\frac{\langle 2345\rangle}{\langle 3456\rangle}, & \alpha_{2}=-\frac{\langle 1234\rangle\langle 3456\rangle}{\langle 2345\rangle\langle 2346\rangle}, & \alpha_{3}=-\frac{\langle 2346\rangle}{\langle 3456\rangle}, & \alpha_{4}=-\frac{\langle 1236\rangle\langle 3456\rangle}{\langle 2346\rangle\langle 2356\rangle}, \\
\alpha_{5}=\frac{\langle 2356\rangle}{\langle 3456\rangle}, & \alpha_{6}=-\frac{\langle 1256\rangle\langle 3456\rangle}{\langle 2356\rangle\langle 2456\rangle}, & \alpha_{7}=-\frac{\langle 2456\rangle}{\langle 3456\rangle}, & \alpha_{8}=-\frac{\langle 1456\rangle}{\langle 2456\rangle},
\end{array}
$$

where we find the letters given by the following 9 Plücker coordinates:

$$
\langle 1234\rangle,\langle 2346\rangle,\langle 2345\rangle,\langle 1236\rangle,\langle 2356\rangle,\langle 1256\rangle,\langle 2456\rangle,\langle 1456\rangle,\langle 3456\rangle \text {. }
$$

Note that these are the $\mathcal{A}$ coordinates of a cluster in $G(4,6): 6$ frozen variables plus 3 unfrozen ones, and they can be arranged as the quiver below. This quiver can be obtained as the dual of the plabic graph [1], and in this case, any square moves on plabic graphs are in 1:1 correspondence with mutations on the cluster. Thus, without explicitly performing square moves, we see the complete alphabet of the Yangian is given by the collection of all $\mathcal{A}$ coordinates, which are the 15 Plücker coordinates.

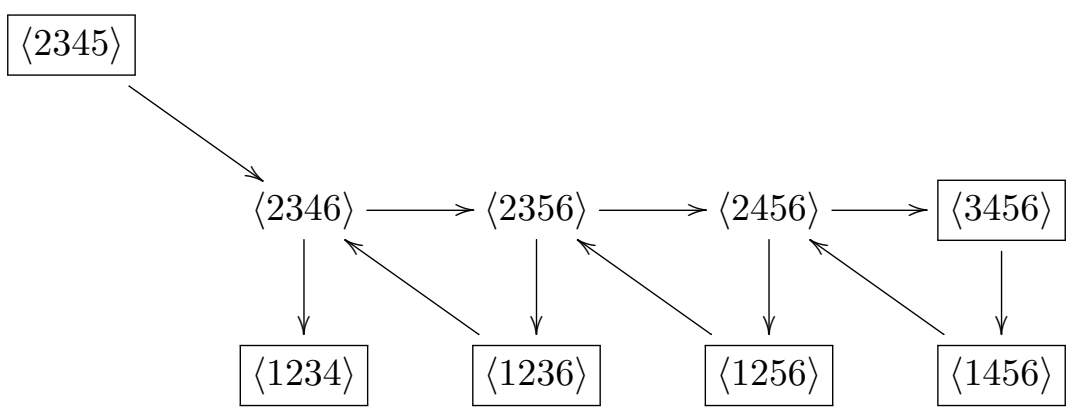


For general $n$, one can always choose a representative plabic graph, which corresponds to an initial cluster of $G(4, n)$ (as a generalization of the quiver above). It is straightforward to see that for such a graph, the letters are given by $4(n-4)+1$ Plücker coordinates, with $n$ frozen variables and $3(n-5)$ unfrozen ones. The square moves still correspond to mutations but only those special ones acting on a node with two incoming and two outgoing arrows (dual to a square face), and such mutations generate new letters that are still Plücker coordinates.

\subsection{Letters of $n=6$ and $n=7$ invariants}

From the discussions above, one can determine the collection of letters for all possible Yangian invariants for $n=6$. In fact, either from the union of alphabets for NMHV invariants or from that of $\mathrm{N}^{2} \mathrm{MHV}$ one, we get $9+6=15 \mathcal{A}$ coordinates of $G(4,6)$.

Now we move to $n=7$, where in addition to NMHV Yangian invariants, we also have two classes of $\mathrm{N}^{2} \mathrm{MHV}$ ones, which are the first cases with letters other than Plücker coordinates. Since $\mathrm{N}^{2} \mathrm{MHV}$ invariants are given by parity-conjugate of the NMHV ones for $n=7$, the poles of the former can be obtained as the parity conjugate of poles of the latter (which are Plücker coordinates). It is straightforward to see that parity conjugate of $\langle i i+1 j j+1\rangle$ and $\langle i-1 i i+1 j\rangle$ are proportional to Plücker coordinates of the same type, thus for $n=7$ the only kind of Plücker coordinates with non-trivial parity conjugate are $\langle 1346\rangle$ and cyclic permutations; under parity, we have $\langle 7(12)(34)(56)\rangle$ etc., and we see that 35 unfrozen cluster variables of $G(4,7)$ appear as poles of Yangian invariants. However, we will see new letters appearing for $\mathrm{N}^{2} \mathrm{MHV}$ invariants, which are not parity conjugate of Plücker coordinates, and they are crucial for getting all cluster variables in this case.

A representative plabic graph of a Yangian invariant in the first class is

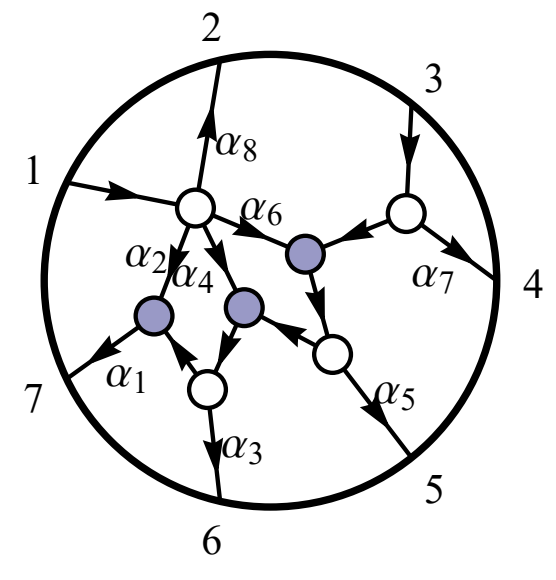

The corresponding $C$ matrix reads

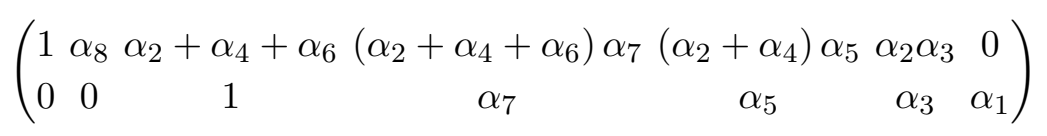


and the solution of $C(\alpha) \cdot Z=0$ is

$$
\begin{aligned}
& \alpha_{1}=\frac{\langle 3456\rangle}{\langle 4567\rangle}, \alpha_{2}=-\frac{\langle 4567\rangle\langle 5(12)(34)(67)\rangle}{\langle 2567\rangle\langle 3456\rangle\langle 3457\rangle}, \alpha_{3}=-\frac{\langle 3457\rangle}{\langle 4567\rangle}, \alpha_{4}=-\frac{\langle 4567\rangle\langle 7(12)(34)(56)\rangle}{\langle 2567\rangle\langle 3457\rangle\langle 3467\rangle}, \\
& \alpha_{5}=\frac{\langle 3467\rangle}{\langle 4567\rangle}, \alpha_{6}=-\frac{\langle 1267\rangle\langle 4567\rangle}{\langle 2567\rangle\langle 3467\rangle}, \quad \alpha_{7}=-\frac{\langle 3567\rangle}{\langle 4567\rangle}, \alpha_{8}=-\frac{\langle 1567\rangle}{\langle 2567\rangle},
\end{aligned}
$$

where we have defined

$$
\langle a(b c)(d e)(f g)\rangle \equiv\langle a b d e\rangle\langle a c f g\rangle-\langle a b f g\rangle\langle a c d e\rangle .
$$

Again since we are interested in polynomials of Plücker coordinates, the letters for this plabic graph are the following 10:

$$
\begin{aligned}
& \langle 1267\rangle,\langle 1567\rangle,\langle 2567\rangle,\langle 3456\rangle,\langle 3457\rangle,\langle 3467\rangle, \\
& \langle 3567\rangle,\langle 4567\rangle,\langle 5(67)(34)(12)\rangle,\langle 7(56)(34)(12)\rangle .
\end{aligned}
$$

By considering cyclic rotations of the labels, we see 7 new letters of the form $\langle i(i i+1)(i+2 i+3)(i-2 i-1)\rangle$ for $i=1, \cdots, 7$, in addition to the $7+28$ Plücker coordinates. However, if we compare to the 42 (unfrozen) $\mathcal{A}$ coordinates of $G(4,7), 7$ variables of the form $\langle i(i-1 i+1)(i+2 i+3)(i-3 i-2)\rangle$ (for $i=1, \cdots, 7)$ are still missing. Can we find such cluster variables as letters of this Yangian invariant?

Very nicely, we will see that by applying square moves, these new letters appear in equivalent plabic graphs for the same Yangian invariant. Recall that it is more convenient to describe the square moves using face variables, which are given by monomials of our coordinates (certain edge variables) [1]. For an internal face with face variable $f$, after a square move, the only new factor introduced in the new set of face or edge variables is given by $1+f$.

We consider square move on either of the two internal faces (the left one is adjacent to $\alpha_{2}, \alpha_{4}$, and the right one is adjacent to $\alpha_{4}, \alpha_{6}$ ); their face variables are

$$
f_{1}=\frac{\alpha_{4}}{\alpha_{2}}=\frac{\langle 3456\rangle\langle 7(12)(34)(56)\rangle}{\langle 3467\rangle\langle 5(12)(34)(67)\rangle}, \quad f_{2}=\frac{\alpha_{6}}{\alpha_{4}}=\frac{\langle 1267\rangle\langle 3457\rangle}{\langle 7(12)(34)(56)\rangle} .
$$

After performing the square move, we obtain the following new factors,

$$
1+f_{1}=-\frac{\langle 3457\rangle\langle 6(12)(34)(57)\rangle}{\langle 3467\rangle\langle 5(12)(34)(67)\rangle}, \quad 1+f_{2}=\frac{\langle 1257\rangle\langle 3467\rangle}{\langle 7(12)(34)(56)\rangle},
$$

and we see that a new letter $\langle 6(12)(34)(57)\rangle$ appears. This letter and its cyclic permutations exactly give the missing 7 cluster variables mentioned above! 
A representative plabic graph of a Yangian invariant in the second class is

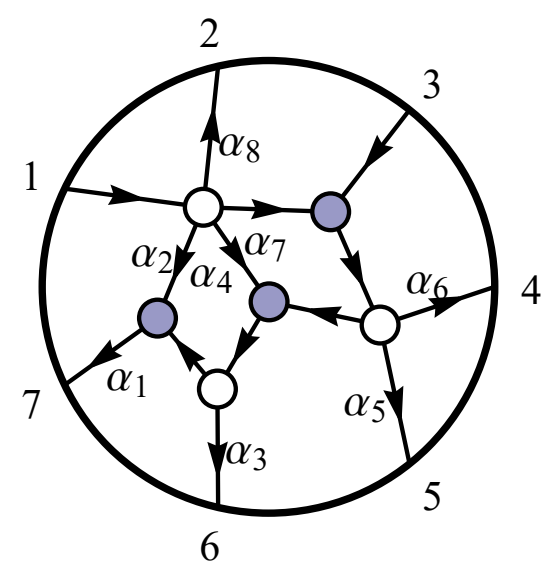

The corresponding $C$ matrix reads

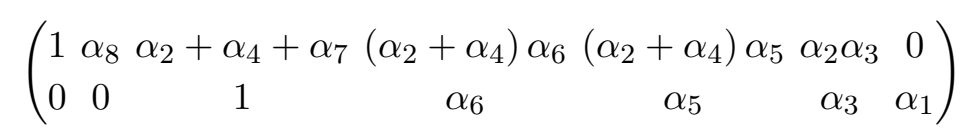

and the solution of $C(\alpha) \cdot Z=0$ is

$$
\begin{array}{ll}
\alpha_{1}=\frac{\langle 3456\rangle}{\langle 4567\rangle}, & \alpha_{2}=-\frac{\langle 4567\rangle\langle 3(12)(45)(67)\rangle}{\langle 2367\rangle\langle 3456\rangle\langle 3457\rangle}, \quad \alpha_{3}=-\frac{\langle 3457\rangle}{\langle 4567\rangle}, \\
\alpha_{4}=-\frac{\langle 1237\rangle\langle 4567\rangle}{\langle 2367\rangle\langle 3457\rangle}, & \alpha_{5}=\frac{\langle 3467\rangle}{\langle 4567\rangle}, \quad \alpha_{6}=-\frac{\langle 3567\rangle}{\langle 4567\rangle}, \quad \alpha_{7}=\frac{\langle 1267\rangle}{\langle 2367\rangle}, \quad \alpha_{8}=-\frac{\langle 1367\rangle}{\langle 2367\rangle} .
\end{array}
$$

We obtain the following letters, which also miss the last class of 7 cluster variables:

$$
\langle 1237\rangle,\langle 1267\rangle,\langle 1367\rangle,\langle 2367\rangle,\langle 3456\rangle,\langle 3457\rangle,\langle 3467\rangle,\langle 3567\rangle,\langle 4567\rangle,\langle 3(12)(45)(67)\rangle \text {. }
$$

Similarly we can apply square moves of either of the following two internal faces (the left is adjacent to $\alpha_{2}, \alpha_{4}$, and the right one is adjacent to $\alpha_{4}, \alpha_{7}$ ) with variables

$$
f_{1}=\frac{\alpha_{4}}{\alpha_{2}}=\frac{\langle 1237\rangle\langle 3456\rangle}{\langle 3(12)(45)(67)\rangle}, \quad f_{2}=\frac{\alpha_{7}}{\alpha_{4}}=-\frac{\langle 1267\rangle\langle 3457\rangle}{\langle 1237\rangle\langle 4567\rangle},
$$

and the new factors obtained from such moves are given by

$$
1+f_{1}=\frac{\langle 1236\rangle\langle 3457\rangle}{\langle 3(12)(45)(67)\rangle}, \quad 1+f_{2}=-\frac{\langle 7(12)(36)(45)\rangle}{\langle 1237\rangle\langle 4567\rangle} .
$$

Thus we find yet another new letter $\langle 7(12)(36)(45)\rangle$, and together with cyclic permutations, they give 7 new variables which are not cluster variables! In fact, by considering all possible plabic graphs for these Yangian invariants, we obtain an alphabet that consists of all 42 unfrozen cluster variables of $G(4,7)$ (plus 7 frozen ones), as well as 14 new letters that are not cluster variables (all from the second type of invariants); 7 in the cyclic class of $\langle 7(12)(36)(45)\rangle$ and 7 in the class of $\langle 7(14)(23)(56)\rangle$. These 63 letters make up the complete alphabet for $n=7$ Yangian invariants. 


\subsection{Algebraic letters of $\mathrm{N}^{2} \mathrm{MHV}$ invariants}

Finally, we present our main example of algebraic letters, namely leading singularities of four-mass boxes (see the left figure below). Without loss of generality, we consider that of the four-mass box $(a, b, c, d)=(1,3,5,7)$ for $n=8$ (the right figure below; the other four-mass box $(a, b, c, d)=(2,4,6,8)$ is given by cyclic rotation by 1$)$.
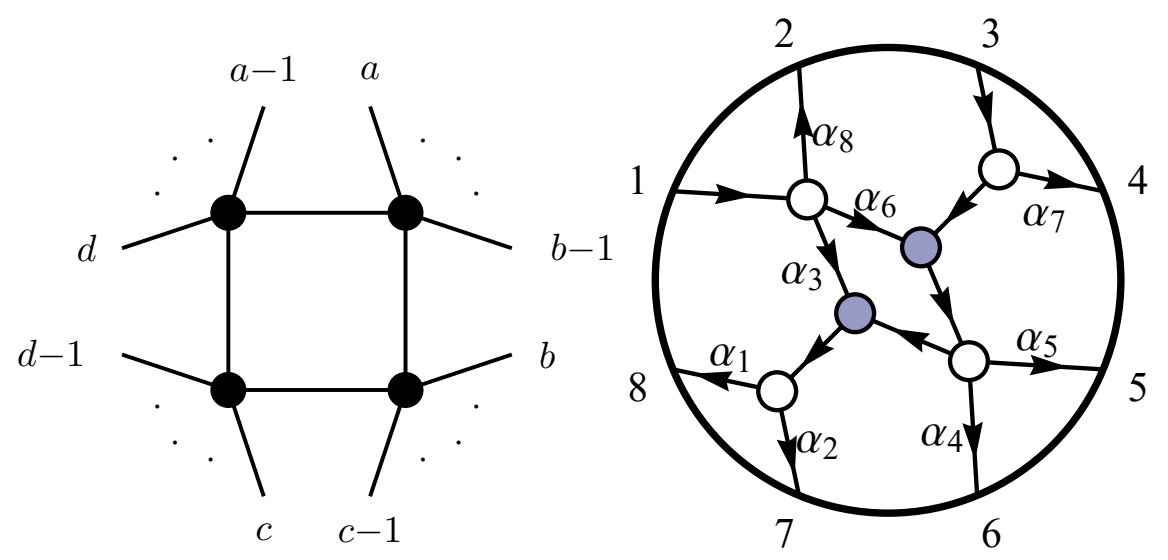

For this representative plabic graph, the corresponding $C$ matrix reads

$$
\left(\begin{array}{cccccccc}
1 & \alpha_{8} & \alpha_{3}+\alpha_{6} & \left(\alpha_{3}+\alpha_{6}\right) \alpha_{7} & \alpha_{3} \alpha_{5} & \alpha_{3} \alpha_{4} & 0 & 0 \\
0 & 0 & 1 & \alpha_{7} & \alpha_{5} & \alpha_{4} & \alpha_{2} & \alpha_{1}
\end{array}\right)
$$

and the solution of $C(\alpha) \cdot Z=0$ is

$$
\begin{array}{llll}
\alpha_{1}=-\frac{\langle 3456\rangle\langle 127 B\rangle}{\langle 456 A\rangle\langle 128 B\rangle}, & \alpha_{2}=\frac{\langle 3456\rangle}{\langle 456 A\rangle}, & \alpha_{3}=-\frac{\langle 456 A\rangle\langle 128 B\rangle}{\langle 3456\rangle\langle 278 B\rangle}, & \alpha_{4}=-\frac{\langle 345 A\rangle}{\langle 456 A\rangle}, \\
\alpha_{5}=\frac{\langle 346 A\rangle}{\langle 456 A\rangle}, & \alpha_{6}=\frac{\langle 1278\rangle}{\langle 278 B\rangle}, & \alpha_{7}=-\frac{\langle 356 A\rangle}{\langle 456 A\rangle}, & \alpha_{8}=-\frac{\langle 178 B\rangle}{\langle 278 B\rangle}
\end{array}
$$

where the two twistors $A, B$ are parametrized as

$$
A=Z_{7}+\frac{\alpha_{1}}{\alpha_{2}} Z_{8}=: Z_{7}+\alpha Z_{8}, \quad B=Z_{3}+\alpha_{7} Z_{4}=: Z_{3}+\beta Z_{4},
$$

then $\alpha, \beta$ satisfy $\langle 12 A B\rangle=0$ and $\langle 56 A B\rangle=0$, i.e.

$$
\alpha=-\frac{\langle 5673\rangle+\langle 5674\rangle \beta}{\langle 5683\rangle+\langle 5684\rangle \beta}, \quad \beta=-\frac{\langle 1237\rangle+\langle 1238\rangle \alpha}{\langle 1247\rangle+\langle 1248\rangle \alpha},
$$

and we have two solutions for these quadratic equations, which we denote as $X_{+}$and $X_{-}$ for $X=A, B$. This is the source of letters involving square roots, and the discriminant of the quadratic equations, $\Delta$, reads

$$
\Delta:=(\langle(34) \cap(127) 568\rangle+\langle(34) \cap(128) 567\rangle)^{2}-4\langle 7(12)(34)(56)\rangle\langle 8(12)(34)(56)\rangle .
$$

All the algebraic letters for this invariant involve the square root of $\Delta$. Therefore, we have the following 2 Plücker coordinates and $8 \mathcal{A}$-like algebraic letters:

$$
\langle 3456\rangle,\langle 1278\rangle,\langle 456 A\rangle,\langle 356 A\rangle,\langle 346 A\rangle,\langle 345 A\rangle,\langle 128 B\rangle,\langle 127 B\rangle,\langle 178 B\rangle,\langle 278 B\rangle \text {. }
$$


For each algebraic letter, one can plug in two solutions, $X_{+}$and $X_{-}$but they are not multiplicative independent since the product is a rational function (which is expected to be given by rational letters from other Yangian invariants), thus we have 8 independent algebraic letters for this plabic graph.

Quite nicely, by a square move on the internal face, we obtain yet another algebraic letter. The face variable of the internal face is

$$
f=\frac{\alpha_{6}}{\alpha_{3}}=-\frac{\langle 1278\rangle\langle 3456\rangle}{\langle 456 A\rangle\langle 128 B\rangle},
$$

and the corresponding square move produces a new factor in the numerator:

$$
1+f=\frac{\langle 456 A\rangle\langle 128 B\rangle-\langle 1278\rangle\langle 3456\rangle}{\langle 456 A\rangle\langle 128 B\rangle}=\frac{\langle(A B) \cap(456) 128\rangle}{\langle 456 A\rangle\langle 128 B\rangle} .
$$

Therefore, from the graph and the one after square move, we find 9 algebraic letters: the 8 in $(2.8)$ and $\langle(A B) \cap(456) 128\rangle$ in $(2.10)$, which are all $\mathcal{A}$-like variables. It is straightforward to check that these 9 algebraic letters generate the same space as the 9 algebraic letters with this $\Delta$ [34], modulo some rational letters. In fact, these 9 letters provide a basis for the algebraic alphabet found in [34].

In fact, we can find all possible algebraic letters for this Yangian invariant by cyclic symmetry. The four-mass box is invariant under cyclic rotation by 2 , and one can apply it to the 8 algebraic letters in (2.8), which take the form $\langle A i j k\rangle$ and $\left\langle B i^{\prime} j^{\prime} k^{\prime}\right\rangle$ for $i, j, k \in$ $\{3,4,5,6\}$ and $i^{\prime}, j^{\prime}, k^{\prime} \in\{7,8,1,2\}$. Under the rotation, we obtain the following 8 letters: $\left\langle A^{\prime} i+2 j+2 k+2\right\rangle,\left\langle B^{\prime} i^{\prime}+2 j^{\prime}+2 k^{\prime}+2\right\rangle$ with

$$
A^{\prime}=Z_{1}+\alpha^{\prime} Z_{2}, \quad B^{\prime}=Z_{5}+\beta^{\prime} Z_{6},
$$

and $\alpha^{\prime}$ and $\beta^{\prime}$ are generated by cyclic rotation by 2 of $\alpha$ and $\beta$ respectively (they share the same square root). Thus, even without performing square moves explicitly, we find 16 distinct algebraic letters (if we rotate by 2 again, no new letters appear); one can check that 9 out of the 16 letters are multiplicative independent.

Alternatively, we can write the algebraic letters similar to cluster $\mathcal{X}$ variables, which are dual conformally invariant. In fact, in the above example, we already see two DCI letters which are internal face variable $f$ and the one after square move, $1+f$. Recall the well-known variables $z$ and $\bar{z}$ defined by the equations:

$$
z \bar{z}=\frac{\langle 1278\rangle\langle 3456\rangle}{\langle 1256\rangle\langle 3478\rangle},(1-z)(1-\bar{z})=\frac{\langle 1234\rangle\langle 5678\rangle}{\langle 1256\rangle\langle 3478\rangle} .
$$

It is easy to see that $f=z /(1-z)$ and $1+f=1 /(1-z)$.

To find $\mathcal{X}$-like letters more systematically, let's consider DCI ratios of the form $f\left(X_{+}\right) / f\left(X_{-}\right)$, where $f(X)$ is any of the $16 \mathcal{A}$-like letters with $X=A, B, A^{\prime}, B^{\prime}$. This is a natural way to find $16 \mathcal{X}$-like letters, which are not multiplicative independent and the rank is 9 as expected. Together with the other half from the box $(2,4,6,8)$, these $\mathcal{X}$-like letters generate precisely the same space of DCI algebraic letters given in [34]. These new 
representation of the same space of algebraic letters are good for studying multiplicative relations, which have been systematically derived and summarized for any multiplicity in [35].

Since this is the only type of $n$-point $\mathrm{N}^{2} \mathrm{MHV}$ algebraic Yangian invariants, we have exhausted algebraic letters for $k=2$ : for each four-mass box $(a, b, c, d)$, there are 9 independent algebraic letters which share the same square root.

\section{Discussions}

In this note, we have proposed an algebraic problem of finding the collection of letters, or arguments of $d$ log's, of any Yangian invariant using parameterizations given by plabic graphs. For a given plabic graph, the $4 k$ polynomial equations $C(\alpha) \cdot Z=0$ provide a map from $4 k$-dimensional cell of $G_{+}(k, n)$, parametrized by $4 k \alpha$ 's, to a collection of functions defined on $G(4, n)$; the alphabet of a Yangian invariant consists of such functions for all plabic graphs related to each other by square moves. In addition to alphabet of NMHV and $\overline{\mathrm{MHV}}$ invariants which are just Plücker coordinates, we show that for $n=6,7$, the union of alphabets for all invariants includes all cluster $\mathcal{A}$ variables of $\mathrm{G}(4, n)$, and for $n=8$ the algebraic letters of $\mathrm{N}^{2} \mathrm{MHV}$ invariants coincide with the 18 multiplicative-independent symbol letters found for two-loop NMHV amplitudes [34].

We have only provided some data for the general problem, and it would be highly desirable to have a general method for constructing letters for any plabic graph, without the need of case-by-case study. This may involve some algorithm directly in $Z$ space, which mimics the construction of cells in $G_{+}(k, n)$ from the map $C \cdot Z=0$ [1]. Such a construction would circumvent the bottleneck of our computation, namely the need to scan through all plabic graphs of a Yangian invariant.

Relatedly, another pressing question here is how to systematically understand the transformations of letters under square moves of the plabic graphs. As we have seen in the top-cell $(\overline{\mathrm{MHV}})$ case, these transformations correspond to certain cluster transformations, and we would like to understand the analog of these for lower-dimensional cells. The appearance of non-cluster variables in the alphabet of certain Yangian invariant is interesting. By studying all possible types of (rational) Yangian invariants with $k=2$ [1], we find that only two of them, the one we have seen above for $n=7$ and one for $n=8$, contain such non-cluster variables. It would be interesting to understand the origin of these non-cluster variables.

Going into fine structures of the alphabet, one can ask "cluster-adjacency" kind of questions not for the poles of a Yangian invariant, but rather the letters which appear in the $d \log$ form. That is, what letters can appear as arguments of a $4 k$-dim $d \log$ form? When the letters are all cluster variables, we expect them to be in the same cluster, but we will need some new ideas when algebraic letters (and those non-cluster variables) are involved. The answer to this kind of questions may provide new insights for the study of symbol alphabet of multi-loop amplitudes.

Last but not least, we would like to study this problem at higher $n$ and $k$. Already for $n=8$, it would be interesting to find the complete alphabet show that the union of alphabets include all the 180 rational letters of [34]. For $n=9$, we find that the $9 \times 9$ 
independent algebraic letters from the $9 \mathrm{~N}^{2} \mathrm{MHV}$ four-mass boxes are not enough for just the two-loop NMHV amplitudes [35]. This is not surprising since there are two types of algebraic Yangian invariants for $k=3$ (both with $\Gamma(C)=2$ ), for which we have not been able to determine the alphabet yet. We leave all these fascinating open questions to future investigations.

Notes added. During the preparation of the manuscript, [36] appeared on arXiv which has some overlap with the result presented in this note.

\section{Acknowledgments}

We are grateful to N. Arkani-Hamed for motivating us to study this problem and for inspiring discussions. We thank Chi Zhang for collaborations on related projects. All the plabic graphs in this paper are made using the Mathematica package positroids.m of J. Bourjaily [37]. This work is supported in part by Research Program of Frontier Sciences of CAS under Grant No. QYZDBSSW-SYS014 and National Natural Science Foundation of China under Grant No. 11935013.

Open Access. This article is distributed under the terms of the Creative Commons Attribution License (CC-BY 4.0), which permits any use, distribution and reproduction in any medium, provided the original author(s) and source are credited.

\section{References}

[1] N. Arkani-Hamed, J.L. Bourjaily, F. Cachazo, A.B. Goncharov, A. Postnikov and J. Trnka, Grassmannian Geometry of Scattering Amplitudes, Cambridge University Press (2016), [DOI] [arXiv: 1212.5605] [INSPIRE].

[2] N. Arkani-Hamed and J. Trnka, The Amplituhedron, JHEP 10 (2014) 030 [arXiv: 1312.2007] [INSPIRE].

[3] L.J. Dixon, J.M. Drummond and J.M. Henn, Bootstrapping the three-loop hexagon, JHEP 11 (2011) 023 [arXiv: 1108.4461] [INSPIRE].

[4] L.J. Dixon, J.M. Drummond, C. Duhr, M. von Hippel and J. Pennington, Bootstrapping six-gluon scattering in planar $N=4$ super-Yang-Mills theory, PoS LL2014 (2014) 077 [arXiv: 1407.4724] [INSPIRE].

[5] L.J. Dixon and M. von Hippel, Bootstrapping an NMHV amplitude through three loops, JHEP 10 (2014) 065 [arXiv:1408.1505] [INSPIRE].

[6] J.M. Drummond, G. Papathanasiou and M. Spradlin, A Symbol of Uniqueness: The Cluster Bootstrap for the 3-Loop MHV Heptagon, JHEP 03 (2015) 072 [arXiv: 1412.3763] [INSPIRE].

[7] L.J. Dixon, M. von Hippel and A.J. McLeod, The four-loop six-gluon NMHV ratio function, JHEP 01 (2016) 053 [arXiv:1509.08127] [INSPIRE].

[8] S. Caron-Huot, L.J. Dixon, A. McLeod and M. von Hippel, Bootstrapping a Five-Loop Amplitude Using Steinmann Relations, Phys. Rev. Lett. 117 (2016) 241601 [arXiv: 1609.00669] [INSPIRE]. 
[9] J. Golden, A.B. Goncharov, M. Spradlin, C. Vergu and A. Volovich, Motivic Amplitudes and Cluster Coordinates, JHEP 01 (2014) 091 [arXiv: 1305.1617] [INSPIRE].

[10] A. Hodges, Eliminating spurious poles from gauge-theoretic amplitudes, JHEP 05 (2013) 135 [arXiv: 0905.1473] [INSPIRE].

[11] S. Caron-Huot, L.J. Dixon, J.M. Drummond, F. Dulat, J. Foster, O. Gürdoğan et al., The Steinmann Cluster Bootstrap for $N=4$ Super Yang-Mills Amplitudes, PoS CORFU2019 (2020) 003 [arXiv : 2005.06735] [inSPIRE].

[12] S. Caron-Huot, L.J. Dixon, F. Dulat, M. von Hippel, A.J. McLeod and G. Papathanasiou, Six-Gluon amplitudes in planar $\mathcal{N}=4$ super-Yang-Mills theory at six and seven loops, JHEP 08 (2019) 016 [arXiv: 1903.10890] [INSPIRE].

[13] L.J. Dixon, J. Drummond, T. Harrington, A.J. McLeod, G. Papathanasiou and M. Spradlin, Heptagons from the Steinmann Cluster Bootstrap, JHEP 02 (2017) 137 [arXiv:1612.08976] [INSPIRE].

[14] J. Drummond, J. Foster, O. Gürdoğan and G. Papathanasiou, Cluster adjacency and the four-loop NMHV heptagon, JHEP 03 (2019) 087 [arXiv: 1812.04640] [INSPIRE].

[15] J.S. Scott, Grassmannians and Cluster Algebras, Proc. Lond. Math. Soc. 92 (2006) 345.

[16] T. Dennen, M. Spradlin and A. Volovich, Landau Singularities and Symbology: One- and Two-loop MHV Amplitudes in SYM Theory, JHEP 03 (2016) 069 [arXiv:1512.07909] [INSPIRE].

[17] T. Dennen, I. Prlina, M. Spradlin, S. Stanojevic and A. Volovich, Landau Singularities from the Amplituhedron, JHEP 06 (2017) 152 [arXiv:1612.02708] [INSPIRE].

[18] I. Prlina, M. Spradlin, J. Stankowicz, S. Stanojevic and A. Volovich, All-Helicity Symbol Alphabets from Unwound Amplituhedra, JHEP 05 (2018) 159 [arXiv:1711.11507] [INSPIRE].

[19] I. Prlina, M. Spradlin and S. Stanojevic, All-loop singularities of scattering amplitudes in massless planar theories, Phys. Rev. Lett. 121 (2018) 081601 [arXiv:1805.11617] [INSPIRE].

[20] S. Caron-Huot and S. He, Jumpstarting the All-Loop S-matrix of Planar N $=4$ Super Yang-Mills, JHEP 07 (2012) 174 [arXiv:1112.1060] [INSPIRE].

[21] J. Drummond, J. Foster, O. Gürdogan and C. Kalousios, Algebraic singularities of scattering amplitudes from tropical geometry, arXiv:1912.08217 [INSPIRE].

[22] N. Henke and G. Papathanasiou, How tropical are seven- and eight-particle amplitudes?, JHEP 08 (2020) 005 [arXiv:1912.08254] [INSPIRE].

[23] N. Arkani-Hamed, T. Lam and M. Spradlin, Non-perturbative geometries for planar $\mathcal{N}=4$ SYM amplitudes, arXiv:1912.08222 [INSPIRE].

[24] D. Speyer and L. Williams, The tropical totally positive Grassmannian, J. Algebr. Comb. 22 (2005) 189.

[25] N. Arkani-Hamed, T. Lam and M. Spradlin, Positive configuration space, arXiv:2003.03904 [INSPIRE].

[26] J. Drummond, J. Foster and O. Gürdoğan, Cluster Adjacency Properties of Scattering Amplitudes in $N=4$ Supersymmetric Yang-Mills Theory, Phys. Rev. Lett. 120 (2018) 161601 [arXiv: 1710.10953] [INSPIRE].

[27] J. Drummond, J. Foster and O. Gürdoğan, Cluster adjacency beyond MHV, JHEP 03 (2019) 086 [arXiv: 1810.08149 ] [INSPIRE]. 
[28] J. Mago, A. Schreiber, M. Spradlin and A. Volovich, Yangian invariants and cluster adjacency in $\mathcal{N}=4$ Yang-Mills, JHEP 10 (2019) 099 [arXiv:1906.10682] [INSPIRE].

[29] J. Golden, A.J. McLeod, M. Spradlin and A. Volovich, The Sklyanin Bracket and Cluster Adjacency at All Multiplicity, JHEP 03 (2019) 195 [arXiv:1902.11286] [INSPIRE].

[30] O. Gürdoğan and M. Parisi, Cluster patterns in Landau and Leading Singularities via the Amplituhedron, arXiv:2005.07154 [INSPIRE].

[31] N. Arkani-Hamed, Positive Geometries in Kinematic Space, lectures at the CMSA workshop, Harvard University, Cambridge, U.S.A., October (2019).

[32] N. Arkani-Hamed, H. Thomas and J. Trnka, Unwinding the Amplituhedron in Binary, JHEP 01 (2018) 016 [arXiv:1704.05069] [INSPIRE].

[33] S. He and C. Zhang, Notes on Scattering Amplitudes as Differential Forms, JHEP 10 (2018) 054 [arXiv: 1807.11051] [INSPIRE].

[34] S. He, Z. Li and C. Zhang, Two-loop Octagons, Algebraic Letters and $\bar{Q}$ Equations, Phys. Rev. D 101 (2020) 061701 [arXiv: 1911.01290] [InSPIRE].

[35] S. He, Z. Li and C. Zhang, The symbol and alphabet of two-loop NMHV amplitudes from $\bar{Q}$ equations, arXiv:2009.11471 [INSPIRE].

[36] J. Mago, A. Schreiber, M. Spradlin and A. Volovich, Symbol alphabets from plabic graphs, JHEP 10 (2020) 128 [arXiv:2007.00646] [INSPIRE].

[37] J.L. Bourjaily, Positroids, Plabic Graphs, and Scattering Amplitudes in Mathematica, arXiv:1212.6974 [INSPIRE]. 